\title{
BABAR TESTS OF LORENTZ AND CPT SYMMETRY
}

\author{
F. MARTíNEZ-VIDAL \\ FOR THE BABAR COLLABORATION \\ IFIC, Instituto de Física Corpuscular, Universitat de València and CSIC, \\ E-46071 Valencia, Spain, and \\ Università di Pisa, Dipartamento di Fisica, Scuola Normale Superiore and \\ INFN, I-56127 Pisa, Italy. \\ E-mail: martinef@slac.stanford.edu
}

\begin{abstract}
Tests of $C P T$ and $T$ symmetries and a limit on the difference between the decay rates of the two mass eigenstates in $B^{0}$-mesons oscillations are reported. The reconstructed $B$ decays, comprising both $C P$ and flavor eigenstates, are obtained from $\Upsilon(4 S) \rightarrow B \bar{B}$ decays collected by the BABAR detector at the PEP-II asymmetricenergy $B$ Factory at SLAC. Sensitivity projections for sidereal time modulation of the $C P T$-violating parameter based on an explicit and general $C P T$-breaking standard model extension are also discussed.
\end{abstract}

\section{Introduction}

The original observation of $C P$ violation in the $B^{0}$-meson system to states like $J / \psi K_{S}^{0} 1,2$ and the current and foreseen high integrated luminosities at $B$ factories have opened the window for more detailed investigations in $B$ meson decays. Among them, those which are receiving today a great deal of experimental and theoretical attention are $C P$ asymmetries and other Unitarity Triangle related measurements, using the large number of different $B$-decay channels (with branching ratios of the order of $10^{-4}-10^{-6}$ ) accessible in these experiments. Nevertheless, these are not the only studies required to fully understand the $C P$ violation mechanism, and more generally the Cabibbo-Kobayashi-Maskawa (CKM) quark-mixing matrix, of the standard model (SM). Moreover, although the mass difference between the $B^{0}$-mass eigenstates is known with high precision ${ }^{3}$, our knowledge of other aspects of the reach phenomenology of $B^{0}$-meson oscillations is meager. In particular, the violation of $C P$ symmetry may include the possibility of $C P T$ violation in oscillation, as well as violation of the $T$ symmetry and a difference between the lifetimes of the mass eigenstates. In addition, an explicit and general CPT-breaking standard model extension (SME) pre- 
dicts that the $C P T$-violating effects, if present, should reveal a dependence with the magnitude and orientation of the $B$-meson momentum and a corresponding variation with sidereal time. All these $C P T$-violating effects could provide clear signature for GUT-scale physics ${ }^{4}$.

\section{Quark-Flavor oscillations in the $B^{0}$ system}

The $B^{0}$-meson system is usually described by the effective Hamiltonian $\mathbf{H}=\mathbf{M}-i \boldsymbol{\Gamma} / 2$, where $\mathbf{M}$ and $\boldsymbol{\Gamma}$ are two-by-two Hermitian matrices describing, respectively, the mass and decay-rate components. Under $C P$ or $C P T$ symmetry, $M_{11}=M_{22}$ and $\Gamma_{11}=\Gamma_{22}{ }^{\text {a }}$. In the limit of $C P$ or $T$ invariance, $\Gamma_{12} / M_{12}=\Gamma_{21} / M_{21}=\Gamma_{12}^{*} / M_{12}^{*}$, so $\Gamma_{12} / M_{12}$ is real. The masses $m_{H, L}$ and decay rates $\Gamma_{H, L}$ of the two eigenstates of $\mathbf{H}$ form the complex eigenvalues $\omega_{H, L} \equiv m_{H, L}-\frac{i}{2} \Gamma_{H, L}$. The light and heavy $B$-meson mass eigenstates are superpositions of $B^{0}$ and $\bar{B}^{05}$,

$$
\begin{aligned}
\left|B_{L}\right\rangle & =p \sqrt{1-\mathrm{z}}\left|B^{0}\right\rangle+q \sqrt{1+\mathrm{z}}\left|\bar{B}^{0}\right\rangle, \\
\left|B_{H}\right\rangle & =p \sqrt{1+\mathrm{z}}\left|B^{0}\right\rangle-q \sqrt{1-\mathrm{z}}\left|\bar{B}^{0}\right\rangle,
\end{aligned}
$$

where

$$
\mathrm{z} \equiv \frac{\delta m-\frac{i}{2} \delta \Gamma}{\Delta m-\frac{i}{2} \Delta \Gamma} \quad, \quad \frac{q}{p} \equiv-\sqrt{\frac{M_{12}^{*}-\frac{i}{2} \Gamma_{12}^{*}}{M_{12}-\frac{i}{2} \Gamma_{12}}},
$$

with the definitions $\delta m \equiv M_{11}-M_{22}, \delta \Gamma \equiv \Gamma_{11}-\Gamma_{22}, \Delta m \equiv m_{H}-m_{L}$ and $\Delta \Gamma \equiv \Gamma_{H}-\Gamma_{L}$. The $C P T$-violating and phase-convention-independent quantity $\mathrm{z}^{5 \mathrm{~b}}$ either vanishes by the $C P T$ theorem, or it depends on the 4 momentum of the $B$ meson ${ }^{4}$, as discussed in Sec. 3. A non-zero value of either $\delta m$ or $\delta \Gamma$ is only possible if both $C P$ and $C P T$ are violated. Assuming $C P T$ invariance $(\delta m=0, \delta \Gamma=0)$, and anticipating $|\Delta \Gamma| \ll \Delta m, \Delta m \approx$ $2\left|M_{12}\right|$ and $\Delta \Gamma \approx 2\left|M_{12}\right| \operatorname{Re}\left(\Gamma_{12} / M_{12}\right)$. Detailed SM calculations ${ }^{6}$ find values for the ratio $\Delta \Gamma / \Delta m$ in the range $-0.2 \%$ to $-0.3 \%$.

A state that is initially $B^{0}\left(\bar{B}^{0}\right)$ will develop a $\bar{B}^{0}\left(B^{0}\right)$ component over time, whose amplitude is proportional to the complex factor $q / p(p / q)$,

$$
\begin{aligned}
& \left|B^{0}{ }_{\text {phys }}(t)\right\rangle=\left[g_{+}(t)+\mathrm{z} g_{-}(t)\right]\left|B^{0}\right\rangle-\sqrt{1-z^{2}} \frac{q}{p} g_{-}(t)\left|\bar{B}^{0}\right\rangle, \\
& \left|\bar{B}^{0}{ }_{\text {phys }}(t)\right\rangle=\left[g_{+}(t)-\mathrm{z} g_{-}(t)\right]\left|\bar{B}^{0}\right\rangle-\sqrt{1-\mathrm{z}^{2}} \frac{p}{q} g_{-}(t)\left|B^{0}\right\rangle,
\end{aligned}
$$

\footnotetext{
a The index 1 indicates $B^{0}$ and 2 indicates $\bar{B}^{0}$.

${ }^{\mathrm{b}}$ The parameter $\mathrm{z}$ is equivalent to $\cos \theta$ in the $D E \theta \phi$ formalism, and to $\delta$ (to leading order) in the $\epsilon, \delta$ (kaon system) formalism, see for example ${ }^{4}$.
} 
where $g_{ \pm}(t)=\left(e^{-i \omega_{H} t} \pm e^{-i \omega_{L} t}\right) / 2$. Invariance under $C P$ or under $T$ requires that $\left|\left\langle B^{0} \mid \bar{B}^{0}{ }_{\text {phys }}(t)\right\rangle\right|=\left|\left\langle\bar{B}^{0} \mid B^{0}{ }_{\text {phys }}(t)\right\rangle\right|$, i.e., $|q / p|=1$. Since the magnitude of $|q / p|^{2} \approx 1-\operatorname{Im} \frac{\Gamma_{12}}{M_{12}}$ is very nearly unity in the SM, this factor is usually assumed to be a pure phase. A detailed SM calculation yields $|q / p|-1=(2.5-6.5) \times 10^{-4} 6$.

At the $\Upsilon(4 S)$ resonance, $B^{0}$ mesons are produced in coherent p-wave pairs. If $A_{1,2}$ and $\bar{A}_{1,2}$ are the amplitudes for the decay of $B^{0}$ and $\bar{B}^{0}$, respectively, to the states $f_{1}$ (at time $t_{1}$ ) and $f_{2}$ (at some later time $t_{2}$ ), then the overall amplitude is given by $A=a_{+} g_{+}(\Delta t)+a_{-} g_{-}(\Delta t)$, where $\Delta t=t_{2}-t_{1}$ is the difference in proper decay times and

$$
\begin{aligned}
& a_{+}=-A_{1} \bar{A}_{2}+\bar{A}_{1} A_{2}, \\
& a_{-}=\sqrt{1-\mathrm{z}^{2}}\left[\frac{p}{q} A_{1} A_{2}-\frac{q}{p} \bar{A}_{1} \bar{A}_{2}\right]+\mathrm{z}\left[A_{1} \bar{A}_{2}+\bar{A}_{1} A_{2}\right] .
\end{aligned}
$$

The decay rate then is ${ }^{5}$

$$
\begin{aligned}
\frac{\mathrm{d} N}{\mathrm{~d} \Delta t} \propto e^{-\Gamma|\Delta t|} & {\left[\frac{\left|a_{+}\right|^{2}+\left|a_{-}\right|^{2}}{2} \cosh \left(\frac{\Delta \Gamma \Delta t}{2}\right) \frac{\left|a_{+}\right|^{2}-\left|a_{-}\right|^{2}}{2} \cos (\Delta m \Delta t)-\right.} \\
& \left.\operatorname{Re}\left(a_{+}^{*} a_{-}\right) \sinh \left(\frac{\Delta \Gamma \Delta t}{2}\right)+\operatorname{Im}\left(a_{+}^{*} a_{-}\right) \sin (\Delta m \Delta t)\right], \quad \text { (5) }
\end{aligned}
$$

where $\Gamma \equiv \frac{1}{2}\left(\Gamma_{11}+\Gamma_{22}\right)$. Note that if the decay $f_{1}$ occurs second, we would need to redefine $\Delta t, a_{+}$and $a_{-}$by interchanging the labels 1 and 2 , which would leave Eq. (5) unaffected. Thus, we can retain the definitions $\Delta t=t_{2}-t_{1}$ and those of Eq. (4). Terms proportional to $(q / p) \bar{A}_{1} \bar{A}_{2}$ and $(p / q) A_{1} A_{2}\left(A_{1} \bar{A}_{2}\right.$ and $\left.\bar{A}_{1} A_{2}\right)$ are associated with decays with net (no net) oscillation. Thus, the phase-convention-independent quantity

$$
\lambda_{f}=\frac{q}{p} \frac{\bar{A}_{f}}{A_{f}}
$$

can be associated to each final state $f$. This is the usual parameter used to characterize $C P$ violation which involves interference between decays with and without net oscillation ${ }^{7}$. If one of the $B$ mesons decay to a $C P$ eigenstate $f_{C P}, \lambda_{C P} \equiv(q / p)\left(\bar{A}_{C P} / A_{C P}\right)$, where $A_{C P}\left(\bar{A}_{C P}\right)$ is the amplitude for $B^{0} \rightarrow f_{C P}\left(\bar{B}^{0} \rightarrow f_{C P}\right)$. $C P$ violation is in this case characterized by $\lambda_{C P} \neq \eta_{C P}$ where $\eta_{C P}= \pm 1$ is the final state's $C P$ eigenvalue. This is the type of $C P$ violation observed in decays like $B \rightarrow J / \psi K_{S}^{0}{ }^{1,2}$. Note that the other possible sources of $C P$ violation contained in $\lambda_{C P}$ are $T$ violation $(|q / p| \neq 1)$ and $\left|\bar{A}_{C P} / A_{C P}\right| \neq 1$ (direct $C P$ violation).

The combined time dependence of $B$ mesons decaying either to flavor $\left(B_{\text {flav }}\right)$ or $C P\left(B_{C P}\right)$ eigenstates provides sensitivity to the full set 
of physical parameters, since they are determined either from different samples, or from different proper time dependence ${ }^{5}$. The sensitivity to $\left(\operatorname{Re} \lambda_{C P} /\left|\lambda_{C P}\right|\right) \operatorname{Re} z$ and $\operatorname{Im} \lambda_{C P} /\left|\lambda_{C P}\right|$ is provided by $B_{C P}$ decays, for which the $\Delta t$ dependence is even for the former $(\cosh (\Delta \Gamma \Delta t / 2) \approx 1$ coefficient $)$ and odd $\left(\sin (\Delta m \Delta t)\right.$ coefficient) for the latter. $B_{\text {flav }}$ decays lack explicit dependence on $\operatorname{Im} \lambda_{C P} /\left|\lambda_{C P}\right|$ and the dependence on Re $\mathbf{z}$ is scaled by the $\sinh (\Delta \Gamma t / 2)$ term, which is small for small $\Delta \Gamma^{\mathrm{c}}$. In contrast, the parameters $|q / p|$ and $\operatorname{Im} z$ (and $\Delta m$ ) are determined by the $B_{\text {flav }}$ decays due to its relative abundance compared to $B_{C P}$ decays, where the former is associated with a $\Delta t$-even distribution and the latter with a $\Delta t$-odd $(\sin (\Delta m \Delta t)$ coefficient) distribution. The sensitivity to $|q / p|$ comes mostly from the $B_{\text {flav }}$ sample since violation of $C P$ and $T$ in mixing leads to a difference between the $B^{0} \rightarrow \bar{B}^{0}$ and $\bar{B}^{0} \rightarrow B^{0}$ oscillation amplitudes proportional to $|q / p|^{4}-1^{8}$. For small values of $\Delta \Gamma / \Gamma$, the determination of $\Delta \Gamma / \Gamma$ is dominated by the $B_{C P}$ sample, since the leading dependence on $\Delta \Gamma$ is linear for $B_{C P}$ decays, while it is quadratic for $B_{\text {flav }}$ states. Moreover, the contribution of $\sinh (\Delta \Gamma t / 2)$ do not depend on whether the other $B$ meson $\left(B_{\text {tag }}\right)$ is identified ("tagged") as $B^{0}$ or $\bar{B}^{0}$, so untagged data can be included to improve the sensitivity to $\Delta \Gamma$. We cannot determine $\Delta \Gamma / \Gamma$ and Rez directly because both occur multiplied by $\operatorname{Re} \lambda_{C P}$ in their dominant contributions to the decay rate.

\section{Sidereal time modulation of $C P T$-violating effects}

Using the SME, a perturbative calculation to leading order returns

$$
\delta m, \delta \Gamma \approx \beta^{\mu} \Delta a_{\mu},
$$

where $\beta^{\mu}=\gamma(1, \vec{\beta})$ is the four-velocity of the $B$-meson state in the laboratory frame, and $\Delta a_{\mu}$ are the $C P T$ - and Lorentz-violating coupling coefficients ${ }^{4}$. Equation (7) reveals that the $C P T$-parameter $\mathrm{z}$ will be affected by the meson-momentum magnitude and orientation in the laboratory frame. As a consequence, since laboratory frame rotates with Earth, if $C P T$-violating effects exist, $\mathrm{z}$ will exhibit a sidereal time dependence after converting to a non-rotating frame. Defining the non-rotating frame to be compatible with celestial equatorial coordinates ${ }^{9}$ with $Z$-axis aligned along the Earth's rotation axis, and taking the laboratory $z$-axis aligned

c Note that a non-zero value of either $\delta m_{d}$ or $\delta \Gamma_{d}$ is only possible if both $C P$ and $C P T$ are violated. 
with the direction of the colliding beams, the expression for $\mathbf{z} \equiv \mathbf{z}(\tilde{t}, \vec{p})$ is found to be ${ }^{4}$

$\mathrm{z} \approx \frac{\gamma}{\Delta m-\frac{i}{2} \Delta \Gamma}\left[\Delta a_{0}+\beta \Delta_{Z} \cos \chi+\beta \sin \chi\left(\Delta a_{Y} \sin \Omega \tilde{t}+\Delta a_{X} \cos \Omega \tilde{t}\right)\right]$,

where $\tilde{t}$ denotes the sidereal time, $\Omega$ the Earth's sidereal frequency and $\chi$ the angle between the laboratory (beam) $z$-axis and the Earth's rotation axis $(\cos \chi=z Z=\cos \theta \cos \phi, \theta$ and $\phi$ being the polar and azimuthal coordinates of the detector with respect to the non-rotating frame). Therefore, in addition to the proper-time dependence, Eq. (5) also contains siderealtime and momentum dependence from $\mathrm{z}(\tilde{t}, \vec{p})$. In deriving Eq. (8) it is assumed that the $B$ mesons move along the $z$-axis with equal momenta, which is a good approximation at $B$ factories operating close the $\Upsilon(4 S)$ resonance. Since the meson decays occur quickly on the sidereal-time scale, we can treat $\tilde{t}$ as a parameter independent of $\Delta t$, and therefore it is appropriate to take $\mathrm{z}$ as independent of $\Delta t$ but varying with $\tilde{t}$. Note that any measurement that ignores the dependence on $\tilde{t}$ and meson momentum eliminates sensitivity to $\Delta a_{X}$ and $\Delta a_{Y}$ components, being only sensitive to an average value of the first two terms of Eq. (8). Thus direct comparison of $C P T$ results must be done carefully since the results are experiment dependent.

\section{The experimental setup}

The PEP-II machine consists of two rings (2.2 $\mathrm{km}$ circumference), one of $9.0 \mathrm{GeV}\left(e^{-}\right)$and one of $3.1 \mathrm{GeV}\left(e^{+}\right)$, housed in the former PEP tunnel, with a single collision point. The machine uses the SLAC linac as injector. Table 1 summarizes some of the most relevant design and achieved parameters of the machine. The high luminosities (instantaneous and integrated) are achieved through strong focusing, high currents, large number of bunches and continuous ("trickle") injection. The asymmetric energy of the beams results in a boost $\beta \gamma$ for the $B$ mesons along the $e^{-}$direction (z-axis) of about 0.56 in laboratory frame. The collider has delivered since the November 1999 until July 2004 a total of $254 \mathrm{fb}^{-1}$ (about 266 million $\Upsilon(4 S) \rightarrow B \bar{B}$ decays), of which $244 \mathrm{fb}^{-1}$ (256 million) have been recorded by $B A B A R$. Current luminosity projections estimate a total integrated luminosity of $1-2 \mathrm{ab}^{-1}$ by the end of the decade. At the single PEP-II collision point is placed the BABAR detector, which is described in detail elsewhere ${ }^{10}$. Figure 1 shows an schematic view of the detector and its components together with their main performances. 
Table 1. PEP-II design and reached main parameters.

\begin{tabular}{l|c|c}
\hline & Design & Reached \\
\hline$E[\mathrm{GeV}] e^{-} / e^{+}$ & $9.0 / 3.1$ & yes \\
$I[\mathrm{~mA}] e^{-} / e^{+}$ & $0.6 / 1.3$ & $0.98 / 1.54(1-05-04)$ \\
Number of bunches & 1658 & 1588 \\
$L\left[\mathrm{~cm}^{-2} s^{-1}\right]$ & $3 \times 10^{33}$ & $9.2 \times 10^{33}(21-05-04)$ \\
$L_{\text {int }}\left[\mathrm{pb}^{-1} /\right.$ day $]$ & 135 & $710.5(24-05-04)$ \\
\hline
\end{tabular}

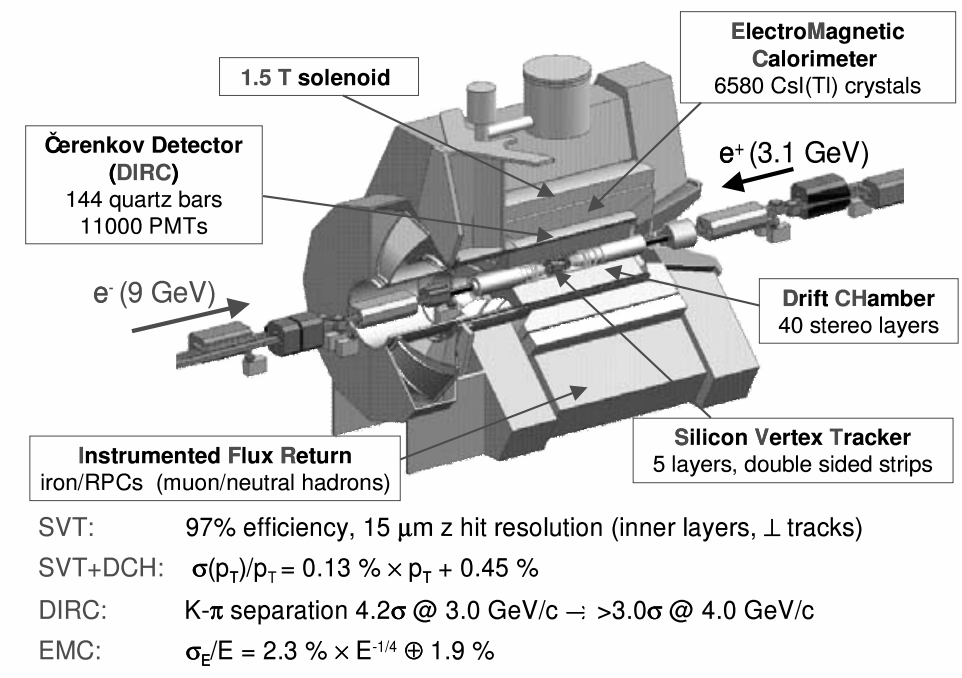

Figure 1. Schematic view of the BABAR detector and its subsystems, together with some of the main performances.

\section{Results with fully reconstructed $B$ decays}

The measurement of $\Delta \Gamma / \Gamma$, and $C P, T$, and $C P T$ violation in mixing has been performed by analyzing $\Upsilon(4 S)$ decays in which one neutral- $B$ meson is fully reconstructed and the other is identified as being either $B^{0}$ or $\bar{B}^{0}$ on the basis of the charges of leptons and kaons, and other indicators of flavor ${ }^{5}$, assuming constant $C P T$-violating parameters. We reconstruct the flavor states ${ }^{\mathrm{d}} B_{\text {flav }}=D^{(*)-} \pi^{+}\left(\rho^{+}, a_{1}^{+}\right)$and $J / \psi K^{* 0}\left(\rightarrow K^{+} \pi^{-}\right)$and the $C P$ eigenstates $B_{C P}=J / \psi K_{S}^{0}, \psi(2 S) K_{S}^{0}, \chi_{c 1} K_{S}^{0}$, and $J / \psi K_{L}^{0}$. The data sample consists of 88 million $\Upsilon(4 S) \rightarrow B \bar{B}$ decays (only about one third of the

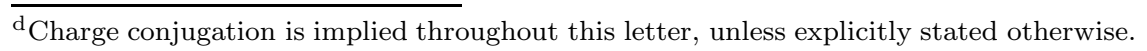


total recorded statistics), which corresponds to approximately $31,000 B_{\text {flav }}$ and 2,600 $C P$ eigenstates. The time interval $\Delta t$ between the two $B$ decays is calculated from the measured separation $\Delta z$ between the decay vertices of $B_{\text {rec }}$ and $B_{\text {tag }}$ along the boost direction $\left(z\right.$-axis) ${ }^{7}, \Delta t \equiv t_{\text {rec }}-t_{\text {tag }} \approx$ $\Delta z /(\beta \gamma c)$.

The measurement requires a precision analysis since competing physics and detector effects that can mimic the behavior we seek must be included ${ }^{5}$. First, the resolution for $\Delta t$ is comparable to the $B$ lifetime and is asymmetric in $\Delta t$. This asymmetry must be well understood lest it be mistaken for a fundamental asymmetry. Second, tagging assigns flavor incorrectly some fraction of the time. Third, interference between weak decays favored by the CKM quark-mixing matrix $\left(b \rightarrow c \bar{u} d\right.$, e.g. $\left.B^{0} \rightarrow D^{-} \pi^{+}\right)$and those doubly-Cabibbo-suppressed (DCS) $\left(b \rightarrow u \bar{c} \bar{d}\right.$, e.g. $\left.\bar{B}^{0} \rightarrow D^{-} \pi^{+}\right)$, roughly proportional to $\left|V_{\mathrm{ub}} V_{\mathrm{cd}}^{*} / V_{\mathrm{cb}}^{*} V_{\mathrm{ud}}\right| \approx 0.02$, has to be explicitly taken into account. Fourth, direct $C P$ violation in the $B_{C P}$ sample could mimic $C P$ violation in mixing and must be parameterized appropriately. Finally, we have to account for possible asymmetries induced by the differing response of the detector to positively and negatively charged particles. To disentangle all these issues we rely mainly on data, making use of a simultaneous maximum-likelihood fit to the time distributions of tagged and untagged, flavor and $C P$ eigenstates. Backgrounds are primarily due to misreconstructed candidates and are studied in data using sidebands. A total of 58 free parameters (32 modeling the signal and 26 the background) are determined with the likelihood fit ${ }^{5}$. The results are

$$
\begin{array}{rr}
\operatorname{sgn}\left(\operatorname{Re} \lambda_{C P}\right) \Delta \Gamma / \Gamma= & -0.008 \pm 0.037 \text { (stat.) } \pm 0.018 \text { (syst.) }[-0.084,0.068], \\
|q / p|= & 1.029 \pm 0.013 \text { (stat.) } \pm 0.011 \text { (syst.) }[1.001,1.057], \\
(\text { dileptons }) & 0.998 \pm 0.006 \text { (stat.) } \pm 0.007 \text { (syst.) }[0.983,1.013], \\
\left(\operatorname{Re} \lambda_{C P} /\left|\lambda_{C P}\right|\right) \operatorname{Re} \mathbf{z}= & 0.014 \pm 0.035 \text { (stat.) } \pm 0.034 \text { (syst.) }[-0.072,0.101], \\
\operatorname{Im} \mathbf{z}= & 0.038 \pm 0.029 \text { (stat.) } \pm 0.025 \text { (syst.) }[-0.028,0.104]
\end{array}
$$

The values in square brackets indicate the $90 \%$ confidence-level (CL) intervals. The measurements of $\operatorname{sgn}\left(\operatorname{Re} \lambda_{C P}\right) \Delta \Gamma / \Gamma$ and $|q / p|$ in the limit when $C P T$ conservation is assumed are unchanged. The largest statistical correlation among the physics parameters appears between $\operatorname{Im} \lambda_{C P} /\left|\lambda_{C P}\right|$ and $\operatorname{Im} z$, which amounts to $17.4 \%$. The second result of $|q / p|$ corresponds to the measurement performed when the two $B$ mesons are reconstructed using high momentum leptons from semileptonic decays (dilepton events) ${ }^{8}$. This measurement constrains $|q / p|$ without assumptions on the value of 
z. Figure 2 (left) shows the results in the $(|q / p|-1,|z|)$ plane, compared to the the SM expectations. If we express the $C P T$ limits as ratios of the $C P T$-violating to the $C P T$-conserving terms we have

$$
\frac{|\delta m|}{m}<1.0 \times 10^{-14}, \quad-0.156<\frac{\delta \Gamma}{\Gamma}<0.042
$$

at the $90 \% \mathrm{CL}$. The parameters $\operatorname{Im} \lambda_{C P} /\left|\lambda_{C P}\right|$ and $\Delta m$ are consistent with recent $B$-factory results ${ }^{1,2,3}$. The value of the $C P$ - and $T$-violating parameter $\operatorname{Im} \lambda_{C P} /\left|\lambda_{C P}\right|$ increases by +0.011 when $C P T$ violation is allowed, which is consistent with the correlations observed in the fit with $C P T$ violation. The value of $\Delta m$ remains unchanged between the two fits. Most contributions to the systematic uncertainties are determined with data and will decrease with additional statistics. The largest single source of uncertainty is the DCS contribution to $\left(\operatorname{Re} \lambda_{C P} /\left|\lambda_{C P}\right|\right) \operatorname{Re} z$ (0.032). This contribution will decrease since with additional statistics a better (and less conservative) treatment of DCS effects will be possible ${ }^{5}$. The $\operatorname{sgn}\left(\operatorname{Re} \lambda_{C P}\right) \Delta \Gamma / \Gamma$ and $|q / p|$ measurements can be used to set constraints on the complex ratio $\Gamma_{12} / M_{12}$, as shown in Fig. 2 (right). Solid contours show the results assuming $\operatorname{Re} \lambda_{C P}>0$ (as expected in the SM based on other experimental constraints), while dashed contours are for $\operatorname{Re} \lambda_{C P}<0$. Inner (outer) contours represent $68 \%$ (90\%) CL regions for two degrees of freedom. The black region shows the predictions of SM calculations when all available experimental inputs are used ${ }^{5}$.
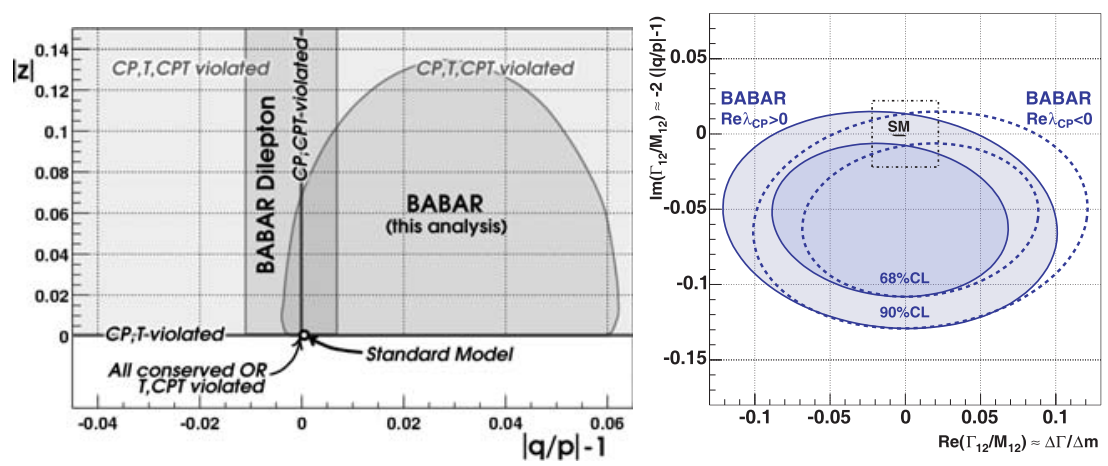

Figure 2. (Left) Favored regions at $68 \%$ CL in the $(|q / p|-1,|z|)$ plane, compared to SM expectation. The axis labels reflect the requirements that both $C P$ and $T$ be violated if $|q / p| \neq 1$ and that both $C P$ and $C P T$ be violated if $|z| \neq 0$. (Right) Constraints at $68 \%$ and $90 \% \mathrm{CL}$ on $\Gamma_{12} / M_{12}$ as determined from the $\operatorname{sgn}\left(\operatorname{Re} \lambda_{C P}\right) \Delta \Gamma / \Gamma$ and $|q / p|$ measurements, compared to SM calculations. 


\section{Sensitivity to $C P T$-breaking sidereal time modulation}

As discussed in Sec. 3 and explicitly shown in Eq. (8), the $C P T$-violating parameter $z$, if non-zero, depends on center-of-mass boost $(\beta \gamma)$, latitude of the collider $(\theta)$ and azimuthal orientation of boost direction $(\phi)$. The approximate values of these parameters for $B A B A R$ (Belle) are estimated to be $0.554,37.42^{\circ} \mathrm{N}$, and $\mathrm{S} 35^{\circ} \mathrm{E}\left(0.425,36.15^{\circ} \mathrm{N}\right.$, and $\left.\mathrm{S} 45^{\circ} \mathrm{E}\right)$, respectively, from which we estimate $\cos \chi=-0.65(-0.57)$ and $\sin \chi=0.76(0.82)$. This implies that Belle's boost direction benefits sidereal time varying terms, but BABAR's boost is larger, enhancing slightly all terms of Eq. (8), as shown in Table 2. In order to estimate the sensitivity to $C P T$-breaking sidereal

Table 2. Sidereal time dependence coefficients of Eq. (8) at $B$ factories.

\begin{tabular}{c|c|c|c}
\hline Term & Coefficient & BABAR & Belle \\
\hline$\Delta a_{0}$ & $\gamma$ & 1.14 & 1.09 \\
$\Delta a_{Z}$ & $\beta \gamma \cos \chi$ & -0.36 & -0.24 \\
$\Delta a_{X} / \Delta a_{Y}$ & $\beta \gamma \sin \chi$ & 0.42 & 0.35 \\
\hline
\end{tabular}

modulation a $C P T$ asymmetry sensitive to z can be defined as ${ }^{4}$

$$
A_{f \tilde{f}}^{C P T}(\Delta t, \tilde{t})=\frac{\Gamma_{f \bar{f}}(\Delta t>0, \tilde{t})-\Gamma_{f \bar{f}}(\Delta t<0, \tilde{t})}{\Gamma_{f \bar{f}}(\Delta t>0, \tilde{t})+\Gamma_{f \bar{f}}(\Delta t<0, \tilde{t})} \approx \frac{-2 \operatorname{Im} z \sin \Delta m \Delta t}{1+\cos \Delta m \Delta t},(9)
$$

only valid to first order in $\mathrm{z}$ and $\Delta \Gamma$, and where $f(\bar{f})$ denotes here a flavor eigenstate and its $C P$ conjugate. Figure 3 shows this asymmetry for dimuon $\left(e^{+} e^{-} \rightarrow \Upsilon(4 S) \rightarrow \mu^{+} \mu^{-}\right)$events as a function of sidereal and Pacific time, using a data sample of about $100 \mathrm{fb}^{-1}$. These events provide a $\Delta t=0\left(A_{f \bar{f}}^{C P T}=0\right)$ benchmark, which can be used to evaluate not only the sensitivity but also detector biases and systematic effects to be used for correcting the signal data. A sensitivity on $A_{f \bar{f}}^{C P T}$ at $10^{-3}$ level translates into a sensitivity on $\mathrm{z}$ of about $10^{-2}$. With full BABAR statistics at the end of the decade, the sensitivity will reach $10^{-3}$.

\section{Summary}

Using one third of the data already recorded by $B A B A R$, we have performed a simultaneous measurement of the difference $\Delta \Gamma / \Gamma$ between the decay rates, and of $C P, T$ and $C P T$ violation in the $B^{0}$-meson system. The limits on $\Delta \Gamma / \Gamma$ and $T$ violation in mixing have reached a precision at the level of $8 \%$ and $1 \%(90 \% \mathrm{CL})$, respectively, largely improving previous results ${ }^{11}$. The $C P T$ measurements, $|\delta m| / m<1.0 \times 10^{-14}$ and $-0.156<\delta \Gamma / \Gamma<0.042$, 

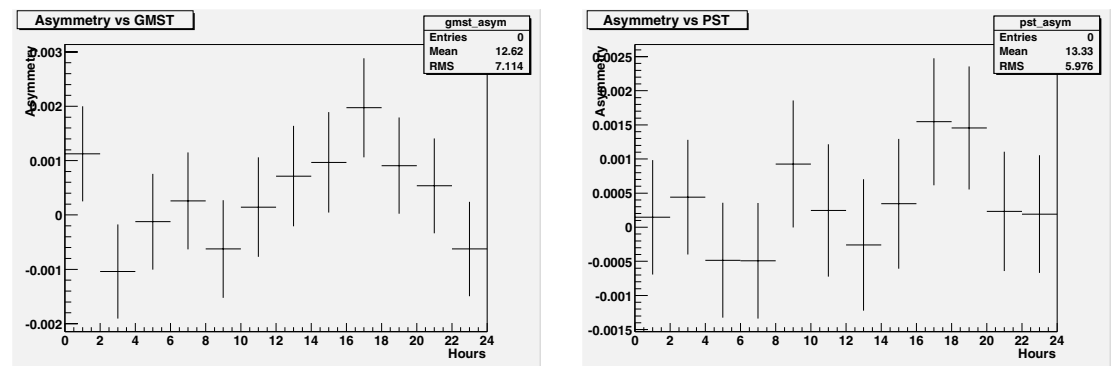

Figure 3. $C P T$ asymmetry $A_{f \bar{f}}^{C P T}(\Delta t, \tilde{t})$ as defined in Eq. (9) for $e^{+} e^{-} \rightarrow \Upsilon(4 S) \rightarrow$ $\mu^{+} \mu^{-}$events as a function of sidereal (left) and Pacific (right) time, from about $100 \mathrm{fb}^{-1}$.

represent the strongest and more general $C P T$ invariance test in the $B^{0}$ system to date ${ }^{12}$. Previous mixing and $C P B A B A R$ measurements performed neglecting these effects are unaffected at this level of precision. We have also discussed within the framework of a general extension of the SM the sensitivity of the $C P T$-violating parameter to sidereal-time modulation. The magnitude and direction of the boost of the PEP-II machine and the latitude of the BABAR detector makes the experiment sensitive to sidereal modulation. The large data sample already recorded and the projections for the forthcoming years will provide the opportunity to perform high precision measurements in $B \bar{B}$ oscillations which may bring surprises.

\section{References}

1. BABAR Collaboration, B. Aubert et al., Phys. Rev. Lett. 89, 201802 (2002).

2. Belle Collaboration, K. Abe et al., Phys. Rev. D 66, 071102(R) (2002).

3. Particle Data Group, S. Eidelman et al., Phys. Lett. B 592, 1 (2004).

4. V.A. Kostelecký, Phys. Rev. D 64, 076001 (2001), and references therein.

5. BABAR Collaboration, B. Aubert et al., Phys. Rev. Lett. 92, 181801 (2004); Phys. Rev. D 70, 012007 (2004).

6. M. Beneke et al., Phys. Lett. B 576, 173 (2003). M. Ciuchini et al., JHEP 0308, 031 (2003). S. Laplace et al., Phys. Rev. D 65, 094040 (2002).

7. BABAR Collaboration, B. Aubert et al., Phys. Rev. D 66, 032003 (2002).

8. BABAR Collaboration, B. Aubert et al., Phys. Rev. Lett. 88, 231801 (2002).

9. M. Pérez-Victoria, Phys. Rev. Lett. 83, 2518 (1999). J.M. Chung, Phys. Lett. B 461, 138 (1999).

10. BABAR Collaboration, B. Aubert et al., Nucl. Instr. Meth. A 479, 1 (2002).

11. DELPHI Collaboration, J. Abdallah et al., Eur. Phys. Jour. C 28, 155 (2003).

12. Belle Collaboration, N.C. Hastings et al., Phys. Rev. D 67, 052004 (2003). OPAL Collaboration, R. Ackerstaff et al., Z. Phys. C 76, 401 (1997). 